\title{
Timeless State of Gravity Possible Solution for Cosmological Constant problem
}

\author{
Ahmed Farag Ali \\ Dept. of Physics, Faculty of Sciences, Benha University, Benha 13518, Egypt. \\ ahmed.ali@fsc.bu.edu.eg
}

\begin{abstract}
We study a localization of gravity through the matching point between noninertial frames and local inertial frames. This localization of gravity is done through defining relative gravitational red-shift. This lead to an emergence of a timeless state in a mathematically consistent way. In this timeless state of gravity, we find a geometric interpretation of the speed of light and mass. The experimental support of the timeless state is the quantum entanglement and internal symmetries that are independent of time. Therefore gravity would be responsible for measurements independent of time including quantum entanglement. Timeless state in that sense explain why do we have gravity/gauge correspondence. We conjecture that the universe emerged from a black hole with a global $S U(3) \times S U(2) \times U(1)$ symmetry on its event horizon that produces gauge fields Electromagnetism, weak and strong nuclear force through localization of this global symmetry. Through the localization in gravity field, the timeless measurements such as spin will be correlated with the varying of timeless measurement which is relative gravitational red-shift. We present a gravitational or geometric interpretation of spin- 0 , spin- 1 and spin- $1 / 2$ states. We present an interpretation on why do we measure matter rather than anti-matter. We found that Higgs scalar field is represented by the gravitational red-shift at every point in the space around the black hole. We derive the numerical value of the cosmological constant that could agree with experimental observations.
\end{abstract}

\section{Localization of gravity}

We assume an existence of Schwarzschild black hole with event horizon. We investigate the gravitational red-shift which is a property of general covariance. To localize gravity, we consider two points $(A$ and $R$ ) in the gravitational field of black hole as shown in the following Fig. (1). Notice here these two points form a triangle that follow a geodesics geometry of the considered black hole when connecting the two points with black hole center. If $R$ and $A$ are far enough from $K$, the triangle become approximately Euclidean triangle. Between these two points $A$ and $R$, there are two possible local gravitational measurements as follows:

1. Relative gravitational red-shift which is represented by the ratio at two different points

$$
\frac{z_{A}}{z_{R}}=\frac{\left(1-\frac{r_{s}}{r_{A}}\right)^{-1 / 2}-1}{\left(1-\frac{r_{s}}{r_{R}}\right)^{-1 / 2}-1}
$$




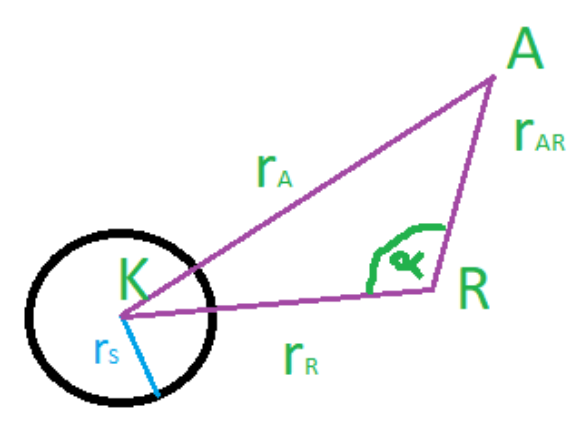

Figure 1: Black hole

2. The difference in gravitational red-shift at two different points.

$$
\Delta z=z_{A}-z_{R}=\left(1-\frac{r_{s}}{r_{A}}\right)^{-1 / 2}-\left(1-\frac{r_{s}}{r_{R}}\right)^{-1 / 2}
$$

\section{Matching point between local gravity measurement and local Inertial frames}

\subsection{Relative gravitational red-shift}

At the moment of change of inertial frames into non-inertial frames, it is mathematically consistent to match the local gravitational measurements with the measurement in local inertial frames. Therefore, we consider the weak gravitational approximations, $r_{s}<<r_{K}$ and $r_{s}<<r_{R}$. The gravitational red-shift for both $A$ and $R$ can be approximated as follows

$$
\begin{aligned}
& z_{A}=\left(1-\frac{r_{s}}{r_{A}}\right)^{-1 / 2}-1 \approx \frac{r_{s}}{2 r_{A}} \\
& z_{R}=\left(1-\frac{r_{s}}{r_{R}}\right)^{-1 / 2}-1 \approx \frac{r_{s}}{2 r_{R}} .
\end{aligned}
$$

We compute the relative gravitational red-shift using $\mathrm{Eq}(1.1)$. We express it in terms of all lengths measured at $R$ including the distance between $A$ and $R\left(r_{A R}\right)$.

$$
\frac{z_{R}}{z_{A}}=\frac{1}{\sqrt{1-\frac{r_{A R}^{2}}{r_{A}^{2}}+2 \frac{r_{R} r_{A R}}{r_{A}^{2}} \cos \alpha}}=\delta
$$

Notice the value of $\alpha$ can be $0 \leq \alpha \leq \pi / 2$. This equation represents the relative gravitational-red-shift between two points $A$ and $R$ in a weak gravitational field. For the case $\alpha=\pi / 2$. The relative gravitational red-shift will be given by

$$
\frac{z_{R}}{z_{A}}=\frac{1}{\sqrt{1-\left(\frac{r_{A R}}{r_{A}}\right)^{2}}}=\delta
$$


On the other side, the measurement in local inertial frames are determined in terms of relative time dilation as follows

$$
\frac{t_{R}}{t_{A}}=\frac{1}{\sqrt{1-\frac{v^{2}}{c^{2}}}}
$$

where $v$ is the relative speed between the two points $A$ and $R$ in the local inertial frames and $c$ is the speed of light.

At the moment of changing inertial frames into non-inertial frames which is matching connecting point between them, the relative gravitational red-shift can set to equal to definition of time dilation in special relativity $\left(1 / \sqrt{1-v^{2} / c^{2}}\right)$ which implies that the ratio $r_{A R}^{2} / r_{A}^{2}$ can be replaced by ratio $v^{2} / c^{2}$. This matching connecting point is legitimate and mathematically consistent since the relative gravitational red-shift introduces a local measurement which should match with special relativity that holds only in "local" inertial frames. Therefore, for mathematical consistency of general relativity, its local measurement should be equivalent to measurement special relativity that hold only in local inertial frames. This means when $\alpha=\pi / 2$, the gamma factor of special relativity emerges as a ratio between the gravitational red-shift at $A$ and $R$. Local gravity measurements depends only on "one variable"; the distance from the gravitational source, which is the reason for velocity ratios turned to be lengths ratios in this delta factor in Eq. (2.4). The ratio $r_{A R}^{2} / r_{A}^{2}$ can be considered as a geometric or gravitational interpretation of the ratio $v^{2} / c^{2}$. This comparison can be written as

$$
\frac{r_{A R}}{r_{A}}=\frac{r_{A R} / t}{r_{A} / t}=\frac{v}{c}
$$

This would support the approach of time varying speed of light as a solution of cosmological puzzles that was suggested in [1]. It may support also the experimental findings of changing physical constants such as fine structure constant in gravitational field as shown recently in [2]. In our case, the ratio $v / c$ is varying depending on the distance from the gravitational source.

We note that time can be inserted easily in the previous equation as a "redundant variable" which can be interpreted that the state of matching local gravity measurements with local inertial frames would correspond to a possible timeless state which is consistent mathematically through the matching process that we performed. The timeless state of universe has been proposed in many contexts such as shape dynamics which introduce a gravitational origin of arrow of time $[3,4]$. The timeless also emerged in Thermal time hypothesis which assume that time only flow in thermodynamics or statistical patterns [5]. It has been mathematically intuited as well that timeless universe is possible [6]

To realize the effect of other values of angle $\alpha$ in weak gravitational field, we consider an approximation which is $r_{A R}<<r_{A}, r_{A R}<<r_{R}$. In that case, the delta factor in Eq. (2.3) is approximated as following

$$
\delta \approx 1-\frac{r_{R} r_{A R}}{r_{A}^{2}} \cos \alpha
$$


It is found that this equation matches with the derivative of Kepler equation.

$$
\frac{d m}{d E}=1-e \cos E
$$

where $m$ is the mean anomaly, $E$ is the eccentric anomaly, and $e$ is the eccentricity. In our approximation, the eccentricity $e$ is approximately equal to $r_{R} r_{A R} / r_{A}^{2}$, and $E$ refers to the angle $\alpha$. This gives a geometric interpretation of Kepler equation from the relative gravitational red-shift.

\subsection{Difference in Gravitational red-shift}

In this section, we compute local measurement as difference in gravitational red-shift. For weak gravitational approximation, we get

$$
\Delta z=z_{R}-z_{A}=\frac{r_{s}}{2 r_{R}}-\frac{r_{s}}{2 r_{A}}
$$

Let us make an approximation as following $r_{A}=r_{R}+x$, where $x<<r_{A}$ and $x<<r_{R}$. In that case, Eq. (2.9) will be rewritten as follows. We use the value of Schwarzschild radius $r_{s}=2 G M / c^{2}$

$$
\Delta z M c^{2}=G M^{2} \frac{x}{r_{R}^{2}}
$$

where $G$, is the gravitational constant, $M$, is the black hole mass and $c$ is the speed of light. From Eq.(2.6), $c$ can be set to equal to $r_{A}$ if we take $t$ to be unity since we agree that $\mathrm{t}$ is a redundant factor through matching local gravity measurement with local inertial frames. We find that Eq. (2.10) can be arranged to take the following form

$$
\Delta z M=\Delta M=G M^{2} \frac{x}{r_{A}^{2} r_{R}^{2}}
$$

where $\Delta M=\Delta z M . \Delta M$ represents relative relation of mass between any two different points in the gravitational field. This would give a geometric representation for relativistic relation $m c^{2}$ in terms of the difference between different points to the black hole. We want to understand the physical meaning of the factor $G M^{2}$ in r.h.s of Eq. (2.11). When we look at Bekenstein-Hawking entropy equation $[7,8]$.

$$
S_{B H}=\frac{c^{3} A}{4 G \hbar}=\frac{4 \pi}{c \hbar} G M^{2}
$$

where $A=16 \pi\left(G M / c^{2}\right)^{2}$ stands for surface area of a black hole. We found that the factor $G M^{2}$ in r.h.s of Eq. (2.11) between any two different points can be expressed in terms of black hole entropy as follows

$$
\Delta z M=\Delta M=\frac{\hbar}{4 \pi} \frac{x}{r_{R}^{2} r_{A}} S_{B H}
$$

We assumed that time is a unity. let us consider this unit as the Planck time. This means that the Planck constant in previous equation can be replaced through the following process 


$$
t_{p}=\sqrt{\frac{\hbar G}{c^{5}}}=1
$$

Since the Planck time is our unity, then c can set to be $r_{A}$. Therefore, the Planck constant in this geometric picture will be given by

$$
\hbar G=r_{A}^{5}
$$

This equation gives a geometric or gravitational interpretation of Planck constant when matching local gravity measurement with local inertial frames. The relative mass between any two different points is therefore given by

$$
\Delta z M=\Delta M=\frac{1}{4 \pi G} \frac{x r_{A}^{4}}{r_{R}^{2}} S_{B H}=\frac{1}{16 \pi G} \frac{x r_{A}^{2}}{r_{R}^{2}} A
$$

We notice that the difference in gravitational red-shift gives an emergence of relative mass. The previous equation gives purely a geometric expression for the relative mass in terms of the gravitational source area of its full entropy. It is experimentally proved that the difference in gravitational potential has an effect on the apparent weight of the $14.4-\mathrm{keV}$ ray of Iron $(\mathrm{Fe})[9,10]$. This may be an experimental support for the derived relation that connect the difference in gravitational red-shift and emergence of mass in this section.

\section{Experimental quantum entanglement evidence of timeless state}

The experimental evidence of the timeless state is the spin measurement in quantum entanglement at two different points in the space [12]. Besides, any internal symmetries of the elementary particles that are measured independent of time. Therefore, it is logically consistent to consider the quantum spin of elementary particles correlated with relative gravitational red-shift between two different points in the space. The same can be applied to all internal symmetries. In that sense, gravity may explain the origin of quantum spin for elementary particles in three different cases as follows:

- The relative gravitational red-shift $z_{R} / z_{A}=1$, would correspond to the spin 1 excitation. In that case, $r_{A}=r_{R}$ which corresponds to isosceles triangle. This state represents the commuting case in spin-statistics in which $z_{R}-z_{A}=z_{A}-z_{R}$.

- The measurement between two different points at different distances from the gravitational source would correspond to the spin-1/2 excitation. In Local inertial frames, this ratio is perfectly $1 / 2$, but it is expected to be varying in strong gravity field as we take gravitational red-shift into consideration of computing this ratio. It can be noted in this state, that $z_{R}-z_{A}=-\left(z_{A}-z_{R}\right)$ which correspond to anti-commutation states in spin-statistics theorem.

- The measurement on the same point, would result spin 0 state. This state is represented by a straight line that has an arbitrary length. This state correspond to the gravitational red-shift at any arbitrary point. 
These spin states can be represnted geometrically as shown in the Fig 2.

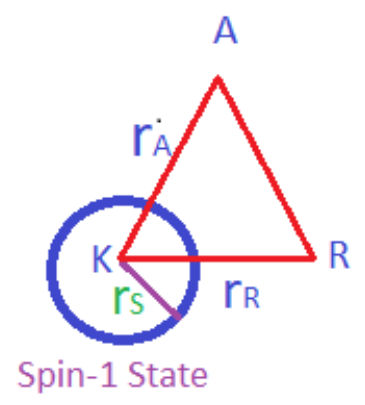

isosceles triangle

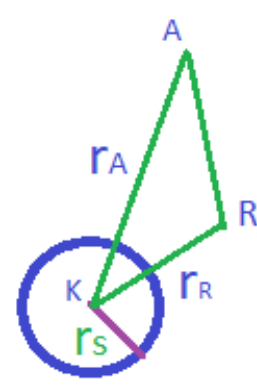

Spin $1 / 2$ state

Scalene triangle

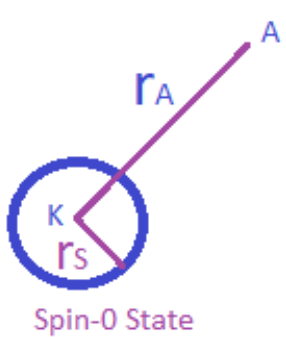

Line segment

Figure 2: Spin States in terms of gravitational red-shift

We interpret that as the quantum spin/internal symmetries as a non-local completeness of local quantum theory which is related to gravitational source. The spin/internal symmetries in that sense may be the gravity effect or gravitational degrees of freedom on every quantum particle. This may open a door for "gravity technology" through the suggested correlation between the quantum spin/internal symmetries and a relative gravitational red-shift in the timeless state.

\section{Gravity and Uncertainty}

In previous sections, we have shown that the concept of velocity is replaced with the relative distance between any any two different points in the space time when we match local gravitational measurements with the local inertial frames. This would generate a timeless state that is mathematically consistent in connecting non-inertial frames with local inertial frames or in other words matching non-locality with locality at their connecting point. In that state, the gravitational measurements happens in terms of only one variable which is the distance from the gravitational source. Time variable at the matching process appear to be a redundant variable. Since time, and therefore velocity dissolve when the matching process happens, therefore there is no meaning to define uncertainty in this timeless state. We conclude that the distance from the gravitational source may form the hidden variable of quantum mechanics, which may complete the connection between quantum mechanics and gravity in one unified theory, which is the timeless state. This may complete the picture that was introduced in EPR [11]. This implies that the uncertainty amount would decrease as the measurement happens closer to the gravitational source. The uncertainty emerges, once we start varying the distance from the black hole. The uncertainty emerges due to the difference in information between point $A$ and point $R$ without knowing the 
distance to the source. This difference is encoded in Eq. 2.16. The difference in information (uncertainty) would be given by

$$
\frac{4 \pi \Delta M r_{R}^{2} r_{A}}{x}=\hbar \quad S_{B H}
$$

Notice that the difference in information between Point $A$ and point $R$ depends on the distances $r_{A}$ and $r_{R}$. If we do not know these values, this difference will be hidden in our local measurements, and therefore uncertainty emerges. Notice that the variables on the left hand side are greater than or equal to the Planck constant. This relation represent the hidden variables which is reason for emergence of uncertainty principle inequality in local measurements.

\section{$5 \quad$ Strong Gravity Case}

In strong gravity case, we can use Eqs. 1.1 and 1.2 without any approximation. These relations can be computed for any two points, and it gives a wide spectrum of measurements of relative gravitational red-shifts and masses in strong gravity field. In strong gravity field, the triangle will not be perfectly Euclidean but can be computed for every kind of measurement by knowing the length of this triangle.

\section{Least computations as a guidance}

The computations of gravitational measurements will use less computations if the relative gravitational red-shift equal to the difference in gravitational red-shift. In that case, what can represent the ratio will certainly represent the difference, with one variable. To achieve the least computations, the local gravitational measurements should satisfy the following condition

$$
z_{A}-z_{R}=\frac{z_{A}}{z_{R}}
$$

For weak gravitational approximation; this condition can be written as

$$
-\frac{r_{s}}{2}=\frac{r_{R}^{2}}{r_{A}-r_{R}}
$$

Or it can be expressed in terms of black hole mass as

$$
G M=-\frac{r_{A}^{2} r_{R}^{2}}{r_{A}-r_{R}}
$$

This equation introduces matter-gravity equation which is noted to be inverse proportionality between Matter and Gravity. Notice the negative sign in Eq. 6.3 where it indicates its a gravitational potential in timeless state. 


\section{Gravity/Gauge Equivalence}

In timeless state, we found that relative gravitational red-shift would explain why do we have timeless measurements such as spin measurements in quantum entanglement. This would support the studies on the correspondence between Gravity and Gauge fields such as Ads/CFT [14]. In the following two sections, we introduce a conjecture of an emergence of universe from a black hole with global symmetry $S U(3) \times S U(2) \times U(1)$ on its event horizon.

\subsection{Standard model as Black hole global Symmetry}

We found that timeless state would justify the timeless measurements such as quantum entanglement and internal symmetries in general. On the other side, the experimental measurements would follow the standard model that has a symmetry $S U(3) \times S U(2) \times$ $U(1)$ where its measurements have been confirmed in local inertial frames [13]. Therefore, we conjecture that our universe emerges from a black hole that has a global symmetry $S U(3) \times S U(2) \times U(1)$ on its event horizon. This group has $12(8+3+1)$ free parameters. Through localization of this symmetry in the gravitational field between any two different points outside the event horizon of the black hole, the gauge fields are generated. In weak gravitational approximation, The timeless measurements such as the spin and internal symmetries in the standard model are correlated with the timeless varying of gravitational red-shift in space. We agree that localization in the gravitational field will be represented by the relative gravitational red-shift.

\section{2 geometric interpretation of Spontaneous Symmetry Break- ing in the timeless state}

The spontaneous symmetry breaking would correspond to the emergence of mass in the timeless state of the universe. As we have shown in previous sections, the mass emerges when when there is a change in gravitational red-shift between two different points at two different distances from the black hole. The black hole has a global symmetry $S U(3) \times$ $S U(2) \times U(1)$ on its event horizon. When we localize this symmetry between two different points, a mass emerges. This localization process would generate a two different spherical symmetry groups $S O(3)$ at the two different points because the gravitational red-shift does not change over any sphere in the space around the black hole. Using isomorphism, $S U(2) / \mathcal{Z}_{2} \sim S O(3)$. This fundamental geometry relation would imply that the $\mathrm{SU}(2)$ symmetry part of the gravity global symmetry has been broken into two different $\mathrm{SO}(3)$ with two different radii, and the mass emerges as a result of difference in value between these two different radii. This means $\mathrm{SU}(3)$ and $\mathrm{U}(1)$ symmetry will remain unbroken which explain why gluons and photon remain mass-less in the standard model and their internal symmetries are measured independent of time such as color and hyper charge. The Higgs modes in that sense can be understood as the gravitational red-shift at every point which formulate the spin-0 modes as we have shown in the previous section. 


\section{Event Horizon as the Edge of Symmetry breaking}

In the symmetry breaking described above, we have shown that black hole horizon works as the edge of at which the global symmetry breaks. This symmetry breaking at the event Horizon between "global" $S U(3) \times \mathrm{SU}(2) \times U(1)$ and "Local" $S U(3) \times \mathrm{SU}(2) \times U(1)$ can explain why do we measure matter rather than anti-matter outside the black hole. This can be understood as follows. If the creation of particle-antiparticle happens at the neighbourhood of event Horizon, then we can call the energy modes that are detected in "Local" $S U(3) \times \mathrm{SU}(2) \times U(1)$ as a Matter or particles, and we can call the Matter or particles that enter the black hole global $S U(3) \times \mathrm{SU}(2) \times U(1)$ as anti-matter or antiparticles. It implies the two different states of matter as follows

- Matter state: It emerges through localization of global symmetry in infinite space around the black hole

- Anti-Matter state: Matter condensed inside a finite space with Schwarzschild radius $r_{s}$

It implies that matter emerges because of the localization of symmetry in infinite space, and antimatter is the condensation in a finite space bounded by Schwarzschild radius. In principle, we cannot measure inside the Schwarzschild radius, We only can measure outside the Schwarzschild radius. This may answer the fundamental question, why do we measure matter and not anti-matter in the universe!

\section{Possible Solution for the Cosmological Constant Prob- lem}

We have shown in previous sections that Spin 0 states are represented by the gravitational red-shift at every point. Therefore, Higgs field is nothing but the gravitational red-shift at every point. Therefore, the Higgs mass would be related to the gravitational red-shift as follows:

$$
z_{0} \propto m_{H} \approx 2.22525 \times 10^{-25} \mathrm{Kg}
$$

where $z_{0}$ is the gravitational red-shift at the scale of our local measurement. We can set $z=K m_{H}$, where $\mathrm{K}$ is a constant that has a dimension $[1 /$ Mass=1/Length $]$ in the timeless state. On the other hand, The cosmological constant can be represented by the vacuum energy which is represented in timeless state by the integration of gravitational red-shift. Therefore, the full integration of $\mathrm{z}$ over its possible varying is

$$
\Lambda=\int_{0}^{K m_{H}} z d z
$$

The integration is performed between the value of $z$ at the event horizon and at the point of measurement, which is the Higgs mass. 


$$
\Lambda=\left.\frac{z^{2}}{2}\right|_{0} ^{K m_{H}}=K^{2} \frac{m_{H}^{2}}{2} \approx K^{2} \times 2.47586878 \times 10^{-50}
$$

The experimental value of cosmological constant is

$$
\Lambda=1.1056 \times 10^{-52} m^{-2}
$$

By comparing Eq. 9.4 with Eq. 9.3, we get the numeric value of the constant $K$ as follows

$$
K=0.06682442081 m^{-1}
$$

In the timeless state, everything is geometry even mass as we shown in the paper. Therefore, the Length unit=Mass unit. $K$ here is understood as a constant with dimension of $1 /$ Length or 1 /Mass in the timeless state. This explanation would suggest that Higgs mass is varying in correlation with the gravitational red-shift

Therefore the Cosmological constant numeric value will be

$$
\Lambda=10^{-52} m^{-2}
$$

The numeric value we derived is around the experimental cosmological constant value [15]. We think this possibly solve the cosmological constant problem when we consider Higgs modes as the gravitational red-shift at every point. This possibly support our approach of studying timeless state.

\section{Mass-Wave Duality}

We notice here that every mass generated between two points in the space, is bounded by two spheres of different radii. Every mass in that sense has a local symmetry of $S U(3) \times$ $U(1) \times S O(3)$. This symmetry introduces a global wave behaviour for every generated mass from the gravity field. This may introduce a gravitational origin on why we have mass-wave duality.

\section{Origin of Conservation of Energy}

Based on gravitational red-shift computations and the timeless state, we can state that for every action and a reaction, there is always a gravitational source to which both events exist simultaneously in a timeless state, which explains the origin of conservation of energy.

\section{Why Timeless State of Gravity is important?}

Time in principle is the change happening for any physical system. To approach a unified picture of physics, we can investigate states in which no change happening, a frozen moment of the physical system. In that state, everything will be reduced to geometry such as spin, mass, color, etc. Timeless state in that sense could introduce a unified picture of different concepts in physics. We hope to evolve this study in the future. 


\section{Conclusion}

We match non-inertial frames with local inertial frames at their connecting point through equating the relative gravitational red-shift with the gamma factor in local inertial frames. We got a timeless state of the universe in which we found a geometric interpretations of speed of light, mass and spin. We found that the measured spin of quantum particles in quantum entanglement is an experimental evidence for the timeless state of the universe, in which relative gravitational red-shift is measured. The spin in that sense is the relative gravitational red-shift between any two different points of measurement. Timeless state implies an equivalency between Gravity and Gauge fields. Based on this equivalency, we conjecture that the black hole has the global symmetry $S U(3) \times S U(2) \times U(1)$ on its event horizon. We present a possible solution for cosmological constant problem value by considering Higgs field as the gravitational red-shift at every point. We hope that the equivalence that we introduce between internal symmetries and gravity would open the door for new gravitational technology.

Acknowledgment The author is deeply grateful for enlightening discussions with Ebtesam Nafie, Klee Irwin, Raymond Aschiem, Hassan Alshal, Bahaa Hany Sorial, Ahmed Al, Fang Fang, Richard Clawson, Marcelo Amaral, David Chester, Haley Irwin, Alexandra Von Waden, Dugan Hammock, and Stephanie Nadanarajah. This work is supported by the quantum gravity research grant, Los Angeles, California.

\section{References}

[1] A. Albrecht and J. Magueijo, Phys. Rev. D 59, 043516 (1999) doi:10.1103/PhysRevD.59.043516 [astro-ph/9811018].

[2] M. R. Wilczynska, J. K. Webb, M. Bainbridge, S. E. Bosman, J. D. Barrow, R. F. Carswell, M. P. Dabrowski, V. Dumont, A. C. Leite, C. Lee, K. Leszczynska, J. Liske, K. Marosek, C. J. Martins, D. Milakovic, P. Molaro and L. Pasquini, [arXiv:2003.07627 [astro-ph.CO]].

[3] J. Barbour, doi:10.1007/978 - 3-0348-0043-313 [arXiv:1105.0183 [gr-qc]].

[4] J. Barbour, T. Koslowski and F. Mercati, [arXiv:1310.5167 [gr-qc]].

[5] A. Connes and C. Rovelli, Class. Quant. Grav. 11, 2899-2918 (1994) doi:10.1088/02649381/11/12/007 [arXiv:gr-qc/9406019 [gr-qc]].

[6] Gisin, N. Mathematical languages shape our understanding of time in physics. Nat. Phys. 16, 114-116 (2020).

[7] J. D. Bekenstein, Phys. Rev. D 7, 2333-2346 (1973) doi:10.1103/PhysRevD.7.2333

[8] S. Hawking, Commun. Math. Phys. 43, 199-220 (1975) doi:10.1007/BF02345020 
[9] R. V. Pound and G. A. Rebka, Jr., Phys. Rev. Lett. 4, 337 (1960). doi:10.1103/PhysRevLett.4.337

[10] R. V. Pound and J. L. Snider, Phys. Rev. Lett. 13, 539 (1964). doi:10.1103/PhysRevLett.13.539

[11] A. Einstein, B. Podolsky and N. Rosen, Phys. Rev. 47, 777-780 (1935) doi:10.1103/PhysRev.47.777

[12] Kwiat, Paul G., et al. "New high-intensity source of polarization-entangled photon pairs." Physical Review Letters 75.24 (1995): 4337.

[13] G. Aad et al. [ATLAS], Phys. Lett. B 716, 1-29 (2012) doi:10.1016/j.physletb.2012.08.020 [arXiv:1207.7214 [hep-ex]].

[14] J. M. Maldacena, Int. J. Theor. Phys. 38, 1113-1133 (1999) doi:10.1023/A:1026654312961 [arXiv:hep-th/9711200 [hep-th]].

[15] P. de Bernardis et al. [Boomerang], Nature 404, 955-959 (2000) doi:10.1038/35010035 [arXiv:astro-ph/0004404 [astro-ph]]. 